\title{
Les podosomes endothéliaux
}

\section{Elisabeth Génot}

> Les podosomes sont des microdomaines d'adhérence formés au niveau de la membrane plasmique de certaines cellules du lignage hématopoïétique. Ils présentent une organisation caractéristique consistant en un cœur d'actine et de protéines associées à sa polymérisation, encerclé d'un anneau constitué de protéines structurales et de signalisation. Outre la présence de marqueurs spécifiques, ils se distinguent des adhérences focales par la présence de métalloprotéases. Des structures apparentées, de composition moléculaire semblable mais d'architecture distincte, les invadopodes, sont formées dans des cellules fibroblastiques ou épithéliales porteuses de l'oncogène $v$-src, ainsi que dans des cellules tumorales à fort potentiel invasif. Une propriété commune aux cellules qui forment des podosomes ou des invadopodes est leur capacité à franchir des barrières anatomiques. Les podosomes ont maintenant été identifiés dans des cellules endothéliales, cellules non hématopoïétiques et non tumorales, dotées de propriétés invasives lorsqu'elles sont engagées dans des processus de néo-vascularisation. Dans ces structures, des spécificités des podosomes et des invadopodes coexistent. L'élucidation de la fonction des podosomes des cellules endothéliales constitue un enjeu majeur en termes de physiopathologie vasculaire. <

Les cellules communiquent en permanence avec leur environnement. La communication entre la matrice et le cytosquelette de la cellule est bidirectionnelle et elle est assurée par des protéines spécialisées, les intégrines. Celles-ci sont le plus souvent localisées au sein de structures particulières, les «contacts focaux », les «adhérences focales » et les «adhérences fibrillaires», identifiés par leur taille, leur forme, leur distribution spatiale et leur composition moléculaire. En outre, ces structures constituent également des plates-formes de signalisation qui

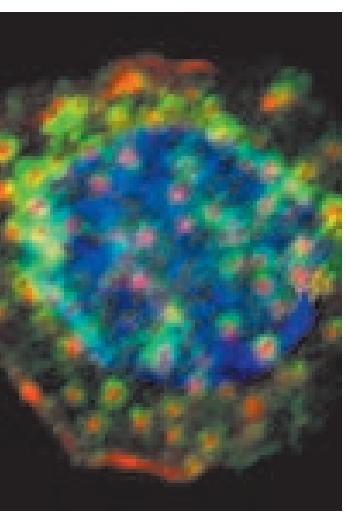

IECB/Inserm U889, 2 , rue Robert Escarpit, 33600 Pessac, France. e.genot@iecb.u-bordeaux.fr

régulent croissance et survie cellulaires. Les podosomes et les invadopodes forment une classe de structures à part et dont la fonction reste à ce jour mal définie. Elles sont beaucoup plus rares, et leur composition moléculaire, leur dynamique, leur architecture et leurs fonctions présumées diffèrent fondamentalement de celles des autres structures d'adhérence membranaires.

\section{Podosomes et invadopodes : deux structures d'actine apparentées}

Les podosomes et les invadopodes sont génériquement regroupés sous le terme PTA (podosome-type adhesion ou plus récemment invadosomes). II s'agit de microdomaines d'adhérence formés au niveau de la membrane plasmique ventrale des cellules et organisés en structures cylindriques à base d'actine [1-3]. Ils regroupent des composants structuraux et des protéines de signalisation comme les adhérences focales mais se distinguent de celles-ci par des marqueurs spécifiques, notamment des éléments de la machinerie de polymérisation de l'actine (Tableau I). Les expériences de vidéomicroscopie ont révélé que ces structures sont extrêmement dynamiques et sont le siège d'une polymérisation/dépolymérisation continuelle de filaments d'actine, procédant perpendiculairement au plan de la cellule. En permanence, les podosomes fusionnent et se dissolvent pour se reformer ensuite (leur demi-vie est de l'ordre de 2 à $20 \mathrm{~min}$ ). Une autre caractéristique est la présence de métalloprotéases autour du cœur d'actine. Initialement découverts dans des fibroblastes transformés par le virus du 


\begin{tabular}{|c|c|}
\hline Cdc42 & GTPase de la famille Rho, impliquée notamment dans la formation des filopodes \\
\hline Complexe Arp2/3 & Complexe de 7 protéines, c'est le moteur de la polymérisation de l'actine \\
\hline WASp ou N-WASp & $\begin{array}{l}\text { Wiskott Aldrich syndrome proteins. Effecteurs de } \mathrm{Cdc42} \text { et directement impliqués dans le processus de } \\
\text { polymérisation de l'actine. WASP est restreint au compartiment hématopoïétique, son homologue N-WASP est } \\
\text { ubiquitaire }\end{array}$ \\
\hline WIP & $\begin{array}{l}\text { WASP-interacting protein. Partenaire de WASp et de N-WASp, module leurs propriétés, affecte notamment le } \\
\text { turnover des podosomes monocytaires (WASp) }\end{array}$ \\
\hline Cortactine ou HSI & $\begin{array}{l}\text { Impliquées dans l'organisation de la polymérisation de l'actine, ces protéines se lient au complexe Arp2/3 et } \\
\text { stabilisent la branche après dissociation de WASp/N-WASp. L'expression de HSl est restreinte au compartiment } \\
\text { hématopoïétique, la distribution de la cortactine est ubiquitaire }\end{array}$ \\
\hline Gelsoline & Elle se fixe aux filaments d'actine et crée une coupure engendrant la dislocation du filament d'actine \\
\hline Dynamine & $\begin{array}{l}\text { GTPase de haut poids moléculaire. En association avec la cortactine et les filaments d'actine, elle permet } \\
\text { la déformation des membranes pour créer des tubules ou des vésicules, en particulier dans les processus } \\
\text { d'endocytose, de morphogenèse et de migration cellulaire }\end{array}$ \\
\hline P190RhoGAP & $\begin{array}{l}\text { Régulateur négatif de l'activité de la GTPase RhoA, qui pourrait réduire les forces contractiles localement ou } \\
\text { moduler la polymérisation de l'actine par les formines }\end{array}$ \\
\hline Métalloprotéases & $\begin{array}{l}\text { Transmembranaires (MT1-MMP), ancrées sur des protéines ou sécrétées (MMP2, MMP9), elles confèrent aux } \\
\text { invadosomes leur fonction de dégradation de la matrice extracellulaire caractéristique. D'autres enzymes pro- } \\
\text { téolytiques telles que la séprase ou l'uPAR (urokinase-type plasminogen activator receptor) n'ont été décrites } \\
\text { qu'associées aux invadopodes }\end{array}$ \\
\hline
\end{tabular}

Tableau I. Les marqueurs des invadosomes. La présence de protéines essentielles et spécifiques distingue les podosomes/invadopodes des autres structures d'adhérence, notamment les acteurs et régulateurs de la polymérisation de l'actine. De très nombreuses autres protéines ont été décrites dans les invadosomes [1].

sarcome de Rous (porteur de l'oncogène $v$-src) dans les années 1980 [4], les podosomes ont ensuite été mis en évidence dans les cellules normales mais dans un nombre restreint de types cellulaires, les cellules dendritiques immatures, les ostéoclastes et les macrophages (Figure IA). Les podosomes des cellules transformées par v-src ont alors été renommés invadopodes, une dénomination qui reflète leur puissante capacité à dégrader la matrice extracellulaire [5]. Les invadopodes ont maintenant été décrits dans certaines cellules tumorales à fort potentiel invasif comme les cellules de mélanomes et de carcinomes mammaires (Figure IB), potentiellement porteuses d'oncogènes. Leur architecture caractéristique (en forme de protrusion sous la cellule, voir Figure 3), leurs propriétés dynamiques (demi-vie d'environ $1 \mathrm{~h}$, plus élevée que celle des podosomes), leur localisation subcellulaire (on les trouve regroupés à proximité de l'appareil de Golgi et du noyau), les distinguent des podosomes des cellules normales. Quelques modèles décrivant l'organisation spatiale des protéines au niveau des podosomes ont été proposés $[1,6]$, alors que l'agencement des composants des invadopodes [7] ne fait pas encore l'objet d'un consensus.

La formation des podosomes des cellules du lignage myélo-monocytaire est dépendante de la matrice, collagène ou fibronectine selon les types cellulaires $[8,9]$, et les podosomes apparaissent en réponse à l'adhérence. Cependant, l'événement premier qui conduit à l'assem- blage des podosomes n'est pas élucidé. Bien que leur apparition semble résulter d'une signalisation "outside-in» au niveau des intégrines apportant l'infrastructure du complexe, la nucléation des filaments opèrerait à partir d'une amorce enrichie en pré-complexe Arp2/3 [10, 11] et/ou cortactine [12, 13], sur lequel convergeraient les signaux des récepteurs à la matrice [14]. Il en va de même pour les invadopodes [15] pour lesquels le rôle de la chaîne $\beta 1$ des intégrines $[16,17]$ et de la cortactine [18] a été décrit. Il est donc possible que podosomes et invadopodes soient élaborés à partir d'une structure-précurseur commune aux cellules normales et tumorales [19].

Plusieurs voies de signalisation contrôlent l'assemblage des deux types de structures: des récepteurs de type tyrosine-kinases et des tyrosine-kinases «nonrécepteur » telles que $\mathrm{Src}$, mais également des membres de la famille des PKC (PKC $\alpha$ et/ou PKC $\delta$ ) [20, 21], la PI3K et des GTPases de la famille Rho $[22,41]$. De plus, hormis les MMP et à l'exception de pl90RhoGAP [23], I'absence de l'un ou l'autre des marqueurs indiqués dans le Tableau / entraîne la disparition des invadosomes. 


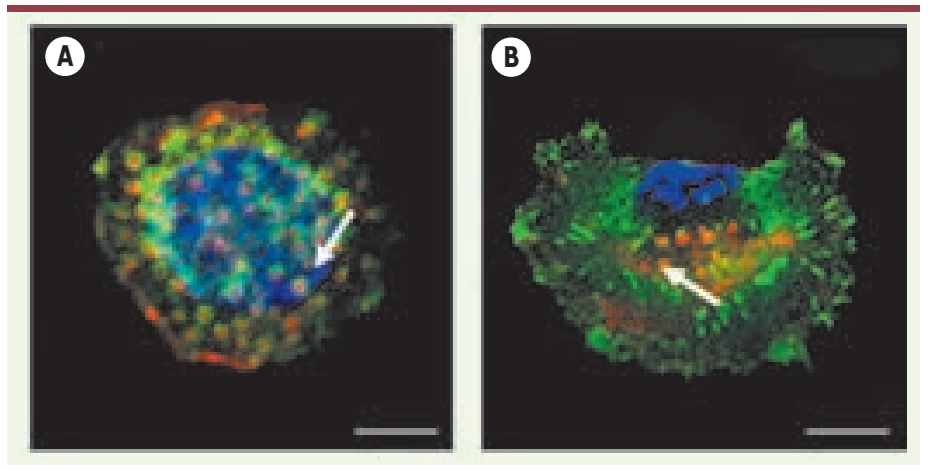

Figure 1. Les podosomes ( $A$ ) et les invadopodes ( $B$ ). Deux entités globalement de même composition moléculaire (voir Tableau 1) mais d'architecture distincte (voir ci-dessous). Visualisation de l'actine polymérisée (rouge) dans les podosomes d'un macrophage humain et dans les invadopodes d'une cellule de la lignée tumorale MDA-MB-231 dérivée d'un carcinome mammaire. La vinculine (vert) est associée aux podosomes et aux invadopodes (marqués par des flèches blanches), mais marque également les adhésions focales qui coexistent avec les invadopodes dans les cellules MDA-MB-231. Le recrutement de la machinerie de polymérisation de l'actine (Arp2/3)

opère à distance de la périphérie et, contrairement aux adhérences focales situées dans le prolongement des fibres de stress, les podosomes ne sont pas en relation directe avec d'autres formations à base d'actine du cytosquelette. Barre $=5 \mu \mathrm{m}$.

\section{Les podosomes des cellules endothéliales}

Plus récemment, les podosomes ont été découverts dans des cellules vasculaires telles que les cellules musculaires lisses et les cellules endothéliales [24]. Dans ces modèles, les podosomes sont inductibles et apparaissent en réponse à des signaux distincts de ceux engendrés par les protéines de la matrice. Cette dernière découverte ouvre un nouveau champ d'investigation pour la biologie des cellules endothéliales car ces cellules qui tapissent la face interne du système cardiovasculaire sont les médiateurs d'échanges privilégiés entre sang et tissus. Elles jouent également un rôle de premier plan dans l'angiogenèse et l'artériogenèse, des programmes dans lesquels les cellules endothéliales acquièrent des capacités invasives et qui conduisent à l'extension du réseau vasculaire. Chez l'adulte, l'angiogenèse naît du bourgeonnement de capillaires existants et est essentiellement limitée à la revascularistion qui accompagne la cicatrisation après une lésion [39]. Beaucoup plus fréquente en pathologie, elle favorise le développement des tumeurs solides en assurant leur irrigation. Elle est aussi à la base de la pathogénicité de rétinopathies et aggrave d'autres maladies telles que la polyarthrite rhumatoïde. L'artériogenèse est le processus de remodelage vasculaire qui survient suite à l'occlusion d'un vaisseau de l'arbre artériel, le plus souvent par un thrombus. Ces processus sont régulés par de très nombreux facteurs parmi lesquels le VEGF (vascular endothelial growth factor) et le TGF- $\beta$ (transforming growth factor) jouent des rôles essentiels.

\section{Le caractère inductible des podosomes endothéliaux}

Nous avons récemment découvert que les cellules endothéliales peuvent former des podosomes en réponse à l'expression de l'oncogène $v$-src [21], un processus décrit dans de nombreux modèles, mais également et plus spécifiquement en réponse à l'expression d'un mutant constitutivement actif de la GTPase Cdc42 [21, 22]. Dans ces cellules, les podosomes peuvent également apparaître après traitement par des agents pharmacologiques tels que les esters de phorbol, révélant la contribution des membres de la famille des PKC [21, 26]. Dans une réponse physiologique, des cytokines sont capables d'induire la formation des podosomes, le TGF- $\beta$ dans des cellules d'origine aortique [27], et le VEGF ou le TNF- $\alpha$ (tumor necrosis factor $\alpha$ ) dans des cellules veineuses [28]. Nous avons montré que, comme les podosomes des autres types cellulaires, les podosomes des cellules endothéliales ont la capacité de dégrader localement la matrice extracellulaire [21, 27].

\section{L'organisation spatiale des podosomes endothéliaux}

L'organisation spatiale des podosomes dans les cellules endothéliales est variable (Figure 2). L'expression du mutant actif de Cdc42 est responsable de la formation de podosomes individuels, répartis sur l'ensemble de la surface ventrale, de façon analogue aux invadopodes induits par l'expression de l'oncogène $v$-src [21] (Figure 2A). À l'inverse, en présence de TGF- $\beta$, les podosomes apparaissent exclusivement organisés en une superstructure de forme circulaire (Figure 2B) [27], réminiscente des rosettes décrites dans les cellules transformées par le virus du sarcome de Rous [29]. Enfin, dans les cellules d'origine veineuse, ces podosomes peuvent se présenter sous de multiples configurations, localisés en arrière du front de migration des cellules motiles ou assemblés en rosettes ou en groupes (Figure 2C). Ces organisations reflètent probablement différents états de la cellule au cours desquels les podosomes pourraient être engagés dans des processus distincts [30]. Les podosomes pourraient assister la migration dans un plan, leur assemblage et dissociation incessants permettant de générer un mode de locomotion plus rapide que celui basé sur les complexes focaux et adhérences focales. Cependant, une telle interprétation des observations est remise en question par des résultats récents, davantage en faveur d'une fonction d'adhérence portée par les podosomes [6, 30-32]. En immobilisant la cellule et en concentrant les activités enzymatiques portées par les 
métalloprotéases, les rosettes de podosomes pourraient, quant à elles, représenter un dispositif cellulaire destiné à former un chemin sous la cellule pour ménager un espace dans lequel celle-ci s'introduit et progresse dans le stroma.

\section{La contribution du lignage cellulaire \\ à la structure des podosomes}

La comparaison des podosomes et des invadopodes révèle des différences notables (résumées sous forme schématique dans la Figure 3). Visualisé par immunofluorescence, l'anneau de protéines encerclant le cœur d'actine des podosomes des cellules hématopoïétiques est bien délimité (Figure IA) alors que cette organisation bipartite apparaît moins distinctement dans les invadopodes (Figure IB), une particularité que l'on retrouve dans les cellules endothéliales (Figure 2), Aussi, les invadopodes se présentent sous forme de projections sous la cellule mais, contrairement à une idée reçue, aucune déformation de la membrane plasmique n'est démontrée pour les podosomes [19]. Alors que les forces transmises par l'adhérence (via les intégrines) contribuent à la formation des podosomes [40], le moteur du processus conduisant à la formation des invadopodes est la dégradation de la matrice [5, 19]. L'organisation des filaments d'actine semble différente dans les deux structures [19]. Enfin, la demi-vie des podosomes est bien inférieure à celle des invadopodes [1-3]. Ces paramètres sont en cours d'étude pour les podosomes des cellules endothéliales.

La formation des podosomes des cellules monocytaires (d'origine hématopoïétique) est induite par l'adhérence à la matrice. En revanche, les invadopodes des cellules transformées sont induits à partir d'un signal intracellulaire ( $v$-src par exemple), dans des cellules qui adhèrent par leurs adhérences focales (Figure 1B). Les podosomes des cellules endothéliales vasculaires sont induits par des cytokines et apparaissent indépendamment de la matrice [27]. Au caractère inductible s'ajoute une autre particularité. Bien que parfois dérivées d'un précurseur mésodermique commun aux lignages hématopoïetique et endothélial, l'hémangioblaste, les cellules endothéliales différenciées des vaisseaux n'ont aucun attribut hématopoïétique. Parmi les protéines caractéristiques des invadosomes, certaines jouent un rôle particulièrement important. C-Src est la tyrosine kinase clé dans les ostéoclastes, Hck dans les cellules monocytaires [33], celle des cellules endothéliales n'est pas identifiée. Les protéines WASp/NWASp (Wiskott-Aldrich syndrome proteins) en tant qu'effecteurs de Cdc42 sont directement impliquées dans le processus de polymé-
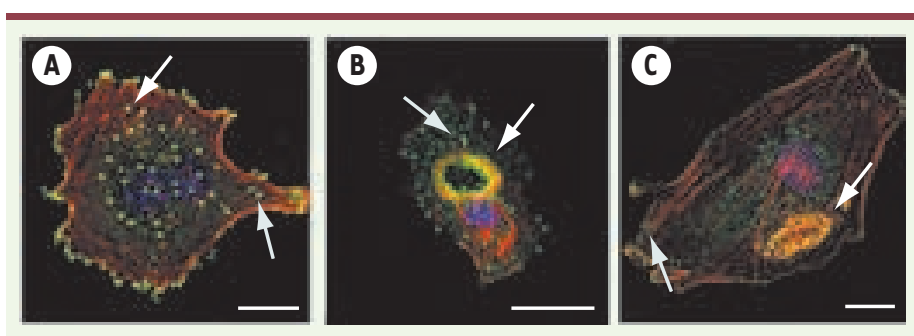

dérivées de cordon ombilical, traitées avec un ester de phorbol). Le marquage vinculine (vert) révèle d'autres types de contacts avec la matrice (contacts focaux et adhésions focales, flèches grises). Barre $=10 \mu \mathrm{m}$.

risation de l'actine. Alors que l'expression de WASP est restreinte au compartiment hématopoïétique, la distribution de son homologue N-WASP est ubiquitaire. Les podosomes des cellules endothéliales sont donc dépendants de N-WASp [24], comme le sont les invadopodes des carcinomes [34]. De même, les protéines HSl (hématopoïétique) et cortactine (ubiquitaire), s'associent aux filaments d'actine pour en stabiliser l'organisation et stimuler leur polymérisation. La kinase Pyk2 (hématopoïétique) et son homologue FAK (focal adhesion kinase) [42] (ubiquitaire) jouent un rôle important pour les podosomes mais sont inégalement représentées dans les cellules hématopoiétiques et endothéliales. Pour ces classes de protéines, l'implication de l'une ou l'autre des isoformes pourrait être à la base de différences architecturales, dynamiques, voire fonctionnelles entre podosomes des cellules hématopoïétiques et des cellules endothéliales, et plus généralement entre podosomes et invadopodes.

À ce jour, podosomes et invadopodes n'ont été décrits qu'in vitro et les critères de définition reposent sur l'observation en deux dimensions. L'endothélium constitue un espace bidimensionnel et cette situation, combinée à la nature inductible des podosomes dans ces modèles, apporte une opportunité unique, celle de pouvoir étudier la formation et la fonction des podosomes endothéliaux in situ, dans un tissu vivant. Ces études sont en cours au laboratoire et permettront d'établir la pertinence des podosomes dans les processus tant physiologiques que pathologiques.

\section{Implication des podosomes dans des processus physio-pathologiques}

Les propriétés des invadosomes, en particulier leur capacité à concentrer en leur sein des intégrines et des métalloprotéases, ont orienté les recherches vers des fonctions d'adhérence, de migration et d'invasion dans l'organisme. Les manifestations physiopathologiques associées à une absence, ou au contraire à l'apparition, d'invadosomes dans plusieurs types cellulaires,

Figure 2. L'agencement des podosomes varie selon l'inducteur et le type de cellule endothéliale. Les podosomes (flèches blanches), déctectés par la colocalisation de l'actine (rouge) et de la vinculine (vert), peuvent être répartis uniformément sur toute la surface ventrale de la cellule ( $\boldsymbol{A}$, cellules aortiques exprimant un mutant constitutivement actif de $\mathrm{Cdc42}$ ), $s^{\prime}$ assembler en une superstructure circulaire ( $B$, cellules aortiques traités par le TGF- $\beta$ ), se regrouper ( $C$, cellules veineuses
vinculine (vert) révèle d'autres types de contacts avec la matrice 
confortent ces hypothèses. Cependant, ces structures peuvent également être observées dans certaines cellules en dehors de ces contextes.

- Les analyses génétiques réalisées à partir des cellules hématopoïétiques de patients atteints du syndrome de Wiskott-Aldrich (WAS), une immunodéficience héréditaire liée au chromosome $X$, ont révélé le rôle fondamental de la protéine WASP dans la formation des podosomes. Les cellules du compartiment hématopoïétique compétentes pour la formation de podosomes et porteuses de mutations perte de fonction, sont dépourvues de podosomes et la pathologie se manifeste par un désordre du trafic de ces cellules dans l'organisme. II reste à définir si l'absence des podosomes est causale ou symptomatique dans ces pathologies.

- Dans les ostéoclastes, la zone de scellement (ZS), une large bande circulaire à base d'actine polymérisée et de protéines associées, globalement de même composition que les podosomes, délimite un compartiment de résorption. Entre deux cycles de résorption, les composants de la zone de scellement se dissocient et l'actine se repolymérise sous forme de podosomes [35]. Les progrès récents dans le domaine ont permis de dissocier la formation des deux entités. Chez la souris, lorsque le gène codant pour Src est invalidé, les ostéoclastes ne présentent pas de zone de scellement mais conservent la capacité d'assembler des podosomes [36]. En l'absence de WIP (WASP-interacting protein) (Tableau I), Ia formation de la zone de scellement est maintenue mais les ostéoclastes deviennent incapables d'assembler des podosomes [6]. Ces déficits sont corrélés à des défauts du remodelage osseux se manifestant par un phénotype d'ostéopétrose (formation osseuse excessive résultant de l'absence du processus de dégradation). L'analyse fonctionnelle des ostéoclastes WIP $P^{-/-}$a permis de préciser le rôle des podosomes dans ce type cellulaire [6] et conduira à une meilleure classification des pathologies osseuses.

- À l'inverse, des cellules de mélanomes, de gliomes, de carcinomes mammaires, de certaines cellules leucémiques, présentent des invadopodes, absents dans leur contrepartie normale (que sont les mélanocytes, les cellules gliales, les cellules épithéliales mammaires et les lymphocytes, respectivement) et qui pourraient rendre compte de leur fort potentiel invasif et métastatique.

- Une situation plus atypique est celle des podosomes qui sont formés en réponse à un stress environnemental. En situation ischémique (condition pathologique où l'apport en oxygène dans les tissus est réduit), les cardiomyocytes réarrangent leur cytosquelette sous forme de podosomes, une adaptation qui pourrait constituer un mécanisme de défense visant à réduire le stress imposé par les forces contractiles de la matrice dans ces situations [37]. À noter que des podosomes ont également été décrits dans des situations de carence nutritive [38].

- Dans le système vasculaire, nous avons détecté des podosomes dans des cellules endothéliales issues d'aortes dilatées, sujettes aux anévrismes et siège d'une néo-vascularisation intense [24]. La recherche de la signification de ces observations fait l'objet de nos recherches.

\section{Perspectives}

Les podosomes peuvent donc être vus comme les éléments moteurs d'un mode de migration trans-tissulaire, d'un dispositif de forage de la matrice extracellulaire, d'un mécanisme de défense, ou encore la manifestation d'une pathologie ou d'un stress. L'étude des mécanismes moléculaires qui président à leur formation et à leur dissociation appa-

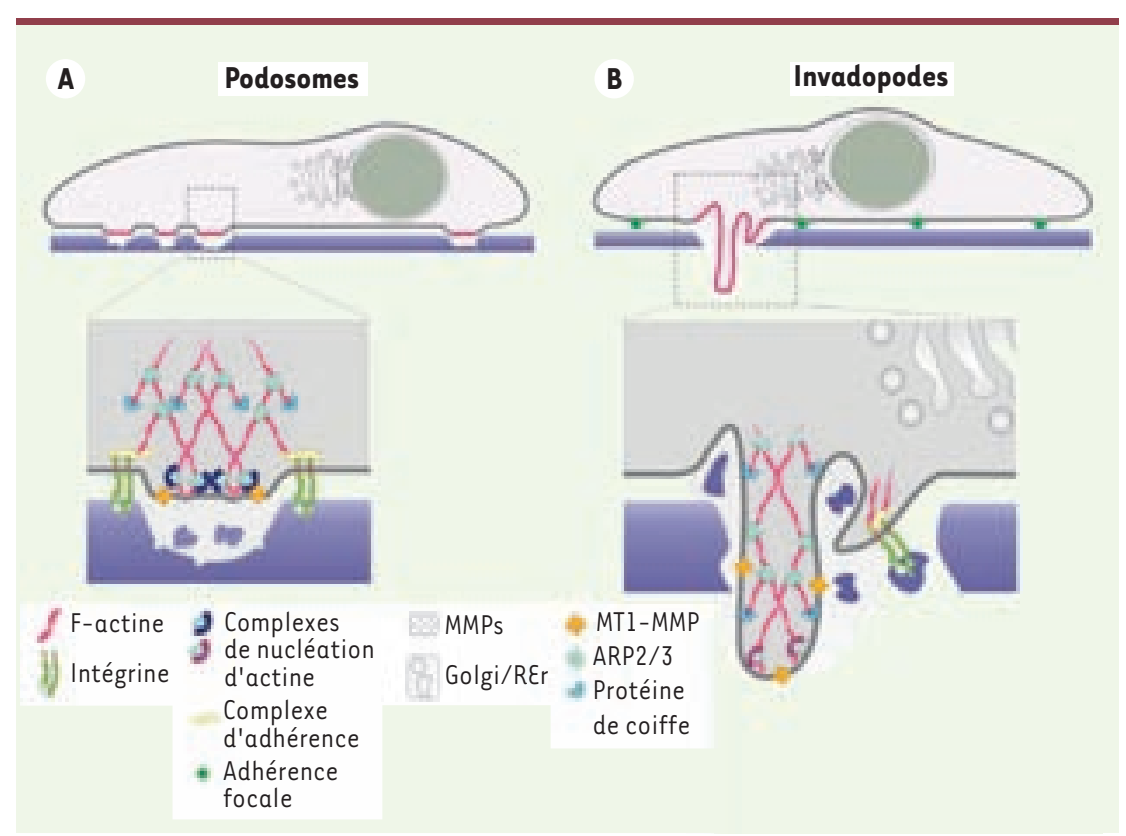

Figure 3. Proposition de représentation schématique d'un podosome de cellule monocytaire (A) et d'un invadopode de cellule transformée (B) mettant en évidence similitudes et différences entre les deux types de structures. L'élongation des filaments d'actine s'effectuerait au niveau du complexe de nucléation localisé à la membrane dans les deux cas. Seul le réseau d'actine branchée dépendant de Arp2/3 est représenté, mais les formines semblent également être impliquées dans l'organisation et la croissance des filaments, au moins dans certains modèles [6]. La nature des composants, l'organisation spatiale et l'efficacité de la dégradation de la matrice diffèrent. II est attendu que les podosomes des cellules endothéliales, non hématopoïétiques et non tumorales, associent des spécificités des podosomes (parce qu'elles sont non tumorales) et des invadopodes (parce qu'elles ne sont pas hématopoïétiques). 
raît ainsi potentiellement porteuse d'avancées importantes dans la compréhension de la biologie des cellules qui forment des invadosomes. La réalisation d'une étude comparative des invadosomes des différents types cellulaires permettra d'identifier les protéines qui gouvernent leurs spécificités et dictent leurs propriétés. À plus long terme, on peut entrevoir la conception de nouvelles stratégies thérapeutiques dans les pathologies osseuses, immunitaires ou vasculaires. Dans le contexte tumoral, l'objectif est le contrôle de la dissémination métastatique qui pourrait être obtenu en agissant non seulement sur le comportement des cellules tumorales mais également sur celui des macrophages et des cellules endothéliales qui participent au processus. $\diamond$

\section{SUMMARY}

\section{The endothelial podosome}

Podosomes are highly dynamic adhesion microdomains formed at the ventral membrane of some monocyte-derived cells. Structurally, their most distinguishing feature is their two-part architecture, consisting in a core of F-actin and actin-associated proteins, surrounded by a ring structure consisting of plaque proteins as well as signalling proteins. In addition to the presence of specific markers, they are distinguished from other adhesion structures by the presence of metalloproteases, endowing them with the ability to degrade the extracellular matrix. Invadopodia are related structures, of similar molecular composition but of distinct architecture, made by fibroblasts or epithelial cells transformed by the $v$-src oncogene or aggressive carcinoma cells. Such membrane-associated cellular devices, now named invadosomes, are thought to have a central role in mediating polarized migration in cells that cross anatomical boundaries. Podosomes have now been shown to form in endothelial cells, non monocytic and non tumoral cells, endowed with tissue invasive activities during vascular remodelling. Here, we summarize the recent advances and developments in this field, discuss how endothelial podosomes combine specificities of monocytic podosomes and invadopodia and provide our provisional outlook into the future understanding of endothelial podosomes. $\diamond$

\section{REMERCIEMENTS}

Nos travaux sont financés par l'Inserm, le ministère de la recherche, la Région Aquitaine, l'ANR, l'ARC, la Fondation de France, la Fondation pour la Recherche Médicale, et La Ligue contre le Cancer. Je remercie tous les membres de l'équipe «signalisation cellulaire » qui ont contribué à découvrir puis à décrire les podosomes des cellules endothéliales et ont ainsi fourni les éléments qui m’ont permis de faire cette synthèse.

rus IMVubohoma consontium Le Consortium Invadosome est un réseau international unissant des laboratoires s'intéressant aux structures d'adhérence impliquées dans les processus invasifs. Il est ouvert à l'ensemble de la communauté scientifique (http://www.invadosomes.org/).

\section{RÉFÉRENCES}

1. Linder S, Aepfelbacher M. Podosomes: adhesion hot-spots of invasive cells. Trends Cell Biol $2003 ; 13: 376-85$.

2. Linder $S$. The matrix corroded: podosomes and invadopodia in extracellular matrix degradation. Trends Cell Biol $2007 ; 3: 107-17$.
3. Buccione R, Orth JD, McNiven MA. Foot and mouth: podosomes, invadopodia and circular dorsal ruffles. Nat Rev Mol Cell Biol 2004 ; $5: 647-57$.

4. David-Pfeuty T, Singer SJ. Altered distributions of the cytoskeletal proteins vinculin and alpha-actinin in cultured fibroblasts transformed by Rous sarcoma virus. Proc Natl Acad Sci USA 1980 ; 77 : 6687-91.

5. Mueller SC, Chen WT. Cellular invasion into matrix beads: localization of beta 1 integrins and fibronectin to the invadopodia. J Cell Sci 1991; $99: 213-25$.

6. Chabadel A, Banon-Rodriguez I, Cluet D, et al. CD44 and beta3 integrin organize two functionally distinct actin-based domains in osteoclasts. Mol Biol Cell 2007 ; 18 : 4899-910.

7. Baldassarre M, Ayala I, Beznoussenko G, et al. Actin dynamics at sites of extracellular matrix degradation. Eur J Cell Biol 2006; 85 : 1217-31.

8. Burns S, Hardy SJ, Buddle J, et al. Maturation of DC is associated with changes in motile characteristics and adherence. Cell Motil Cytoskeleton $2004 ; 57: 118-32$.

9. Sabri S, Foudi A, Boukour S, et al. Deficiency in the Wiskott-Aldrich protein induces premature proplatelet formation and platelet production in the bone marrow compartment. Blood 2006; 108: 134-40.

10. Fan L, Di Ciano-Oliveira C, Weed SA, et al. Actin depolymerizationinduced tyrosine phosphorylation of cortactin: the role of Fer kinase. Biochem J 2004 ; 380 : 581-91.

11. Gunst SJ. Actions by actin: reciprocal regulation of cortactin activity by tyrosine kinases and F-actin. Biochem J $2004 ; 380:$ e7-8.

12. Webb BA, Eves R, Mak AS. Cortactin regulates podosome formation: roles of the protein interaction domains. Exp Cell Res 2006; 312:760-69.

13. Burgstaller $G$, Gimona M. Actin cytoskeleton remodelling via local inhibition of contractility at discrete microdomains. J Cell Sci 2004 ; $117: 223-31$.

14. Luxenburg C, Geblinger D, Klein $\varepsilon$, et al. The architecture of the adhesive apparatus of cultured osteoclasts: from podosome formation to sealing zone assembly. PLoS One $2007 ; 2$ : el79.

15. Ayala I, Baldassarre M, Giacchetti G, et al. Multiple regulatory inputs converge on cortactin to control invadopodia biogenesis and extracellular matrix degradation. J Cell Sci 2008 ; 121 : 369-78.

16. Nakahara H, Mueller SC, Nomizu M, et al. Activation of betal integrin signaling stimulates tyrosine phosphorylation of p190RhoGAP and membrane-protrusive activities at invadopodia. J Biol Chem 1998 ; $273: 9-12$.

17. Mueller SC, Ghersi G, Akiyama SK, et al. A novel protease-docking function of integrin at invadopodia. J Biol Chem 1999; $274: 24947-52$.

18. Bowden ET, Barth M, Thomas D, et al. An invasion-related complex of cortactin, paxillin and PKCmu associates with invadopodia at sites of extracellular matrix degradation. Oncogene $1999 ; 18: 4440$-9.

19. Gimona M, Buccione R, Courtneidge SA, Linder S. Assembly and biological role of podosomes and invadopodia. Curr Opin Cell Biol 2008; $20: 235-41$

20. Hai CM, Hahne P, Harrington $\varepsilon 0$, Gimona M. Conventional protein kinase $\mathrm{C}$ mediates phorbol-dibutyrate-induced cytoskeletal remodeling in $\mathrm{A} 7 \mathrm{r} 5$ smooth muscle cells. Exp Cell Res $2002 ; 280: 64-74$.

21. Tatin F, Varon C, Génot $\varepsilon$, Moreau V. A signalling cascade involving PKC, $\mathrm{Src}$ and $\mathrm{Cdc} 42$ regulates podosome assembly in cultured endothelial cells in response to phorbol ester. J Cell Sci 2006 ; 119 : 769-81.

22. Chellaiah MA, Biswas RS, Yuen D, et al. Phosphatidylinositol 3,4, 5 -trisphosphate directs association of Src homology 2-containing signaling proteins with gelsolin. J Biol Chem 2001 ; 276 : 47434-44.

23. Guegan, F, Tatin F, Leste-Lasserre T, et al. p190B RhoGAP regulates endothelial-cell-associated proteolysis through MT1-MMP and MMP2. J Cell Sci 2008 ; $121: 2054-61$.

24. Moreau V, Tatin F, Varon C, Génot $\varepsilon$. Actin can reorganize into podosomes in aortic endothelial cells, a process controlled by $\mathrm{Cdc} 42$ and RhoA. Mol Cell Biol $2003 ; 23: 6809-22$.

25. Moreau V, Tatin F, Varon C, et al. Cdc42-driven podosome formation in endothelial cells. EurJ Cell Biol $2006 ; 85: 319-25$.

26. Billottet $C$, Rottiers $P$, Tatin $F$, et al. Regulatory signals for endothelial podosome formation. Eur J Cell Biol 2008 ; $87: 543-54$

27. Varon C, Tatin F, Moreau V, et al. Transforming growth factor beta induces rosettes of podosomes in primary aortic endothelial cells. Mol Cell Biol $2006 ; 26: 3582-94$.

28. Osiak AE, Zenner $G$, Linder $S$. Subconfluent endothelial cells form podosomes downstream of cytokine and RhoGTPase signaling. Exp Cell Res 2005 ; $307: 342-53$. 
29. Tarone G, Cirillo D, Giancotti FG, et al. Rous sarcoma virus-transformed fibroblasts adhere primarily at discrete protrusions of the ventral membrane called podosomes. Exp Cell Res 1985; 159 : 141-57.

30. Baron R. L'ostéoclaste et les mécanismes moléculaires de la résorption osseuse. Med Sci (Paris) $2001 ; 17: 1260-69$.

31. van Helden SF, Krooshoop DJ, Broers KC, Raymakers RA, et al. A critical role for prostaglandin $\varepsilon 2$ in podosome dissolution and induction of highspeed migration during dendritic cell maturation. J Immunol 2006 ; $177:$ 1567-74.

32. Nobile C, Lind M, Miro F, et al. Cognate CD4+ T-cell-dendritic cell interactions induce migration of immature dendritic cells through dissolution of their podosomes. Blood 2008 ; 111 : 3579-90.

33. Cougoule C, Carreno S, Castandet J, et al. Activation of the lysosomeassociated p6lHck isoform triggers the biogenesis of podosomes. Traffic $2005 ; 8: 682-94$

34. Lorenz $\mathrm{M}$, Yamaguchi $\mathrm{H}$, Wang $\mathrm{Y}$, et al. Imaging sites of $\mathrm{N}$-WASp activity in lamellipodia and invadopodia of carcinoma cells. Curr Biol 2004 ; $14: 697-703$.

35. Saltel F, Destaing 0, Bard F, et al. Apatite-mediated actin dynamics in resorbing osteoclasts. Mol Biol Cell 2004 ; 15 : 5231-41.
36. Destaing 0, Sanjay A, Itzstein C, et al. The Tyrosine Kinase Activity of c-Src regulates actin Dynamics and organization of podosomes in osteoclasts. Mol Biol Cell 2008; 19: 394-404.

37. VanWinkle WB, Snuggs M, Buja LM. Hypoxia-induced alterations in cytoskeleton coincide with collagenase expression in cultured neonatal rat cardiomyocytes. J Mol Cell Cardiol 1995; $27: 2531-42$.

38. Vesely P, Blase C, Matouskova $\varepsilon$, Bereiter-Hahn J. Arising podosomal structures are associated with neoplastic cell morphological phenotype induced by the microenvironment. Anticancer Res $2006 ; 26: 967-72$

39. Suchting $S$, Freitas $C$, Eichmann A. L'angiogenèse passe sous contrôle du couple Delta-Notch. Med Sci (Paris) $2007 ; 23: 347-8$.

40. Lehoux $S$, Tedgui A. Bases cellulaires de la mécanotransduction dans la cellule endothéliale. Med Sci (Paris) $2004 ; 20: 551-6$.

41. Primeau M, Lamarche-Vane N. Coup d'œil sur les petites GTPases Rho. Med Sci (Paris) 2008; 24 : 157-62.

42. Cornillon J, Campos L, Guyotat D. Focal adhesion kinase (FAK), une protéine aux fonctions multiples. Med Sci (Paris) $2003 ; 19: 743-52$.

TIRÉS À PART

દ. Génot

\section{Collection SCIENCE ET BIOMÉDECINE}

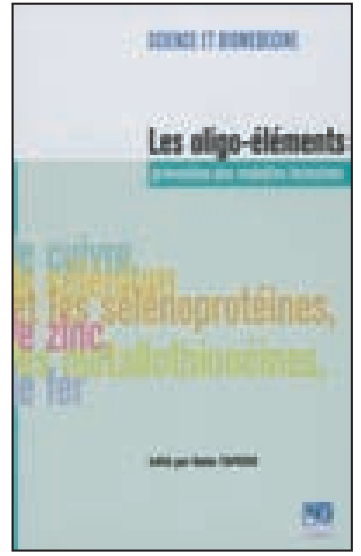

ISBN : 2-84254-107-3 64 pages

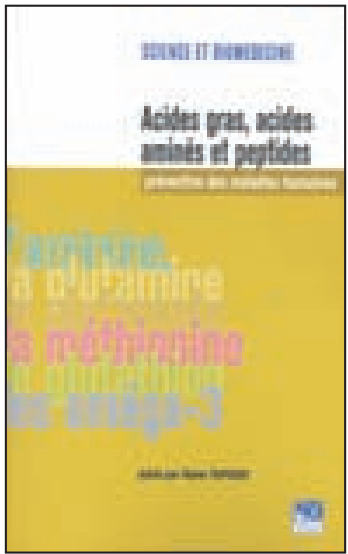

ISBN : 2-84254-108-1 80 pages

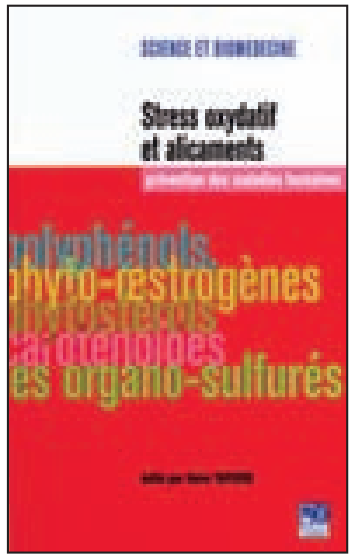

ISBN : 2-84254-111-1 86 pages

\section{Bon de commande}

À retourner à EDK, 2, rue Troyon - 92316 Sèvres Cedex

Tél. : 0155641393 - Fax : 0155641394 - E-mail : edk@edk.fr

NOM :

Prénom :.

Adresse :

Code postal :

Ville :

Pays :

Fonction :

Je souhaite recevoir l'ouvrage Les oligo-éléments : $10 €+3 €$ de port $=\mathbf{1 3} €$ TTC

Je souhaite recevoir l'ouvrage Acides gras, acides aminés et peptides : $12 €+3 €$ de port $=\mathbf{1 5} €$ TTC

Je souhaite recevoir l'ouvrage Stress oxydatif et alicaments : $14 €+3 €$ de port $=17 €$ TTC

$\square$ exemplaire, soit un total de

$\square$ Par chèque, à l'ordre de $\mathbf{E} \mathbf{D} \mathbf{K}$

Par carte bancaire :

$\square$ Visa $\square$ Eurocard/Mastercard

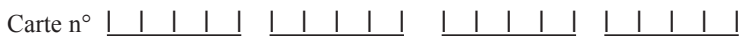

Date d'expiration :

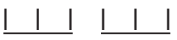

$\mathrm{N}^{\circ}$ de contrôle au dos de la carte : 